\section{The new orthogenesis?}

\author{
Life's Solution: Inevitable Humans in a Lonely \\ Universe \\ Simon Conway Morris \\ Cambridge University Press, Cambridge, UK; 2003. 464 pp. \\ $£ 18.95$, hardback. ISBN 0-521-82704-3.
}

Heredity (2004) 93, 316. doi:10.1038/sj.hdy.6800536

Reviewed by PD Taylor

In what must be one of the most controversial volumes written about evolution in recent years by a respected biologist, Simon Conway Morris here develops an idiosyncratic view of evolution and the emergence of humankind. His thesis is fired by disagreements with the late Stephen J Gould on the role of contingency in evolution, and is underpinned by a degree of theological conviction seldom made so explicit in a scientific book. According to Conway Morris, the appearance of a sentient species with the capability of perceiving and feeling things was an evolutionary inevitability. Furthermore, he contends that our own planet may be unique in providing conditions suited to the appearance of sentient life and we should not therefore hold our breaths in anticipation of being visited by intelligent beings from far-flung corners of the Universe. At the very heart of Conway Morris's thesis is the pervasiveness of evolutionary convergence. The fact that even the most peculiar adaptations have cropped up over and over again during the long history of life on Earth leads the author to suppose that what has evolved was effectively predictable from the outset.

The first part of Life's Solution deals with the improbability of life originating at all. According to Conway Morris, the Earth could be alone among planets in providing exactly the right conditions for life. Our home planet resides in a solar system that may have few counterparts elsewhere in the cosmos, is just the right size, possesses a moon that lends stability to the Earth's axis of rotation, and is shielded from cometary impacts by the 'gatekeeper' planet Jupiter. The core of the book describes the natural histories of an extraordinary plethora of evolutionary convergences, some well known but others less familiar. For example, most biologists will be aware of the convergence between the lens-bearing eyes of vertebrates and cephalopod molluscs, but fewer may know that a similar eye has also evolved in alciopid polychaetes, or that arthropod-like compound eyes have evolved convergently in sabellid polychaetes and some bivalve molluscs. By concentrating on the convergences between groups that are patently not closely related, Conway Morris largely sidesteps the need to introduce phylogeny into his arguments - homoplasy cannot clearly explain the convergence between ants farming fungus and humans farming crops. Surprisingly, for a book on evolution there is not a cladogram in sight.
It is with the evolutionary emergence of mammals, and in particular humans, that Conway Morris ventures into deeper waters. The role for mammalian evolution of the end Cretaceous mass extinction, when the last of the dinosaurs disappeared, is downplayed by Conway Morris. In his opinion, this pivotal event only delayed the rise of mammals because global cooling during the Cenozoic would have favoured mammals over reptiles anyway. Once mammals were in the ascendency, hominids and humans were inevitable in Conway Morris's view: 'If we hadn't walked out of Africa, then probably sooner, rather than later, our analogues would have strolled out of South America, holding tools, and probably enjoying the taste of meat.' (p. 268).

The final major chapter entitled 'Towards a theology of evolution' does not sit well with the rest of the book. Here Conway Morris asserts that theology might 'tell us things about the world that are not only to our real advantage, but will never be revealed by science.' ( $p$ 316). While hostile to 'creation science', Conway Morris nevertheless believes that the sense of purpose possessed by humans and the fact that we inhabit a Universe 'strangely' well suited to our existence are congruent with a Creation.

There are similarities between Conway Morris's thesis and orthogenesis, an evolutionary paradigm in vogue around the turn of the 19th century. Orthogenesis placed great store on the existence of parallel evolutionary trends and, like Conway Morris, viewed evolutionary trajectories as effectively programmed at an early stage. In contrast to the supporters of orthogenesis, who generally attributed evolutionary predetermination to the action of mysterious internal factors, with Natural Selection playing little or no role, Conway Morris is a committed selectionist. Rather than speaking in terms of the 'deeply unfashionable' evolutionary trends of orthogenesis, he prefers to employ the vocabulary of adaptive landscapes, with lineages climbing unerringly towards adaptive peaks.

Skilfully written, Life's Solution is certainly an entertaining read. There is much to admire about Conway Morris's scholarship, which extends well beyond his specialist expertise in palaeontology. His thesis is, however, unconvincing. To Conway Morris, the evolution of life is full of inherencies. Few biologists would deny that the evolutionary pathways have not been entirely random, but this is a far cry from claiming that the attainment of particular endpoints, not least a sentient species like ourselves, was inevitable. There have been just too many unpredictable turns and unexpected obstacles along the evolutionary road.

PD Taylor

Department of Palaeontology, The Natural History Museum, Cromwell Road, London SW7 5BD, UK E-mail: p.taylor@nhm.ac.uk 